\title{
Multiscale simulation of thin-film lubrication: Free-energy-corrected coarse graining
}

\author{
Z.-B. $\mathrm{Wu}^{1, *}$ and X. C. Zeng ${ }^{2, \dagger}$ \\ ${ }^{1}$ LNM, Institute of Mechanics, Chinese Academy of Sciences, Beijing 100190, China \\ ${ }^{2}$ Department of Chemistry, University of Nebraska-Lincoln, Lincoln, Nebraska 68583, USA \\ (Received 7 November 2013; revised manuscript received 27 June 2014; published 4 September 2014)
}

\begin{abstract}
The quasicontinuum method was previously extended to the nonzero temperature conditions by implementing a free-energy correction on non-nodal atoms in coarse-grained solid systems to avoid the dynamical constraint, [Diestler, Wu, and Zeng, J. Chem. Phys. 121, 9279 (2004)]. In this paper, we combine the extended quasicontinuum method and an atomistic simulation to treat the monolayer film lubrication with elastic (nonrigid) substrates. It is shown that the multiscale method with the coarse-graining local elements in the merging regions between the atomistic and continuous descriptions of the substrates can reasonably predict the shear stress profile, the mean separation curve, and the transverse stress profile in the fully atomistic simulation for the tribological system. Moreover, when the nonlocal elements are placed in the merging regions, the inhomogeneous solid atoms in the near regions covered by the cut-off circles of the nonlocal elements replace the homogeneous ones at the equilibrium configuration for the free-energy correction on the non-nodal atoms. The treatment can cause an unphysical sliding between the near and far regions of the upper substrate. It is shown that if the free-energy correction on the non-nodal atoms in the coarse-grained merging regions is removed, the multiscale method can still well reproduce the shear stress profile, the mean separation curve, and the transverse stress profile obtained from the fully atomistic simulation for the system.
\end{abstract}

DOI: 10.1103/PhysRevE.90.033303

PACS number(s): 02.70.-c, 61.20.Ja, 46.55.+d

\section{INTRODUCTION}

Thin-film lubrication systems, which involve the relative motion of two solid substrates separated by a thin fluid film, play a prominent role in tribological processes related to nature, engineering, and social life [1]. On the one hand, although the surfaces may appear to be perfectly flat and smooth on a macroscopic scale, they actually make molecular contacts at relatively few points of asperity, which may lead to plastic deformations. The fluid molecules may diffuse in the interface, as well as be adsorbed and adhered to the solid surfaces. On the other hand, when the solid substrates under a load have a relative displacement, the solid atoms merely oscillate with small amplitude about their equilibrium positions. Due to the microscopic molecular motions at a few points in the interface, far regions of the solid substrates may be slightly removed from the interface. However, the removed regions of the solid substrates, in fact, have multiple spatial scales, which influence the microscopic molecular motions in the tribological systems. The frictional force therefore not only depends on the microscopic movement of both fluid and solid about these points of asperity, but also on the deformation of the far regions of the solid substrates. It is therefore necessary to know both the molecular motion at the interface and the effects of elastic deformation in the extended regions of the solid substrates. It is a challenge to melt the atomistic description required near the interface with the continuum description in the remote regions, in order to obtain a self-consistent global description of the solid substrates.

During the past two decades several effective methods to combine the atomistic and the continuum descriptions of the mechanical behaviors (e.g., crack propagation) in

\footnotetext{
*wuzb@lnm.imech.ac.cn

†xzeng1@unl.edu
}

defective crystals were developed [2]. In particular, with the quasicontinuum (QC) technique [3], a solid is coarsely divided by covering the crystal lattice with a finite-element mesh to treat the quasistatic evolution of defects at zero temperature. A series of theoretical analyses and numerical simulations on this subject were carried out since then [4-21]. On the one hand, from the point of view of the kinetic evolution of solids at a nonzero temperature, a finite temperature version of the QC method was developed. On the other hand, many theoretical results were obtained with multiscale modeling, including effects of ghost force, a priori error estimates and atomistic-continuum adaptivity. In particular, the present authors extended the QC method to nonzero temperature conditions and applied it to treat the thin-film lubrication, which are summarized below to show the objectives of this paper. First, to handle kinetic processes in solids at a nonzero temperature, a dynamically constrained extension of the QC method was designed [22]. The atoms underlying the elements are assumed to move in lock step with the nodes, so the thermal vibrations of those atoms are frozen. For a two-dimensional (2D) crystal of Lennard-Jonesium, the error of the isotropic stress in the solid of coarse grains depends linearly on the degree of coarse division and on the absolute temperature. Then, in order to avoid the above error generated by using the dynamically constrained QC method, an alternative approach for the free-energy function of the coarse-grained system was proposed [23]. For a two-dimensional crystal of LennardJonesium, the isotropic stress is in good agreement with the fully atomistic result, independent of both the degree of coarse division and the absolute temperature. Finally, in an important application, the dynamically constrained QC method is extended to treat processes involving fluid-solid interfaces $[24,25]$. For shear stress profiles, the dynamically constrained hybrid atomistic coarse-graining (HACG) method yields results in excellent agreement with the fully atomistic results. Only the mean separation distance for the dynamically 


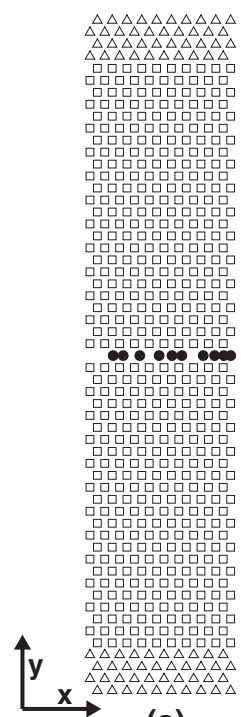

(a)

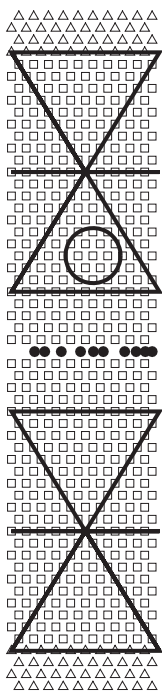

(b)

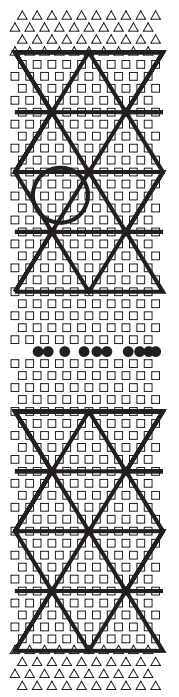

(c)
FIG. 1. Schematic diagram of idealized two-dimensional tribological system with periodic boundary conditions in $x$ direction at temperature $T=0 \mathrm{~K}$ and register $\alpha=0$. (a) Fully atomistic description; (b) coarse graining of far regions of substrates with local elements (a cut-off circle is embedded itself in a representative element); (c) coarse graining of far regions of substrates with nonlocal elements (a cut-off circle of a representative element is surrounded by several nearby elements). The fluid atoms are marked by solid circles, the solid atoms in the substrates are marked by hollow squares, and the wall atoms are marked by hollow triangles.

constrained HACG simulation is shorter than that for the fully atomistic simulation, which can be thought as due to the dynamic constraints on the underlying atoms in the coarsegrained far regions. However, when the free-energy-corrected QC method with local elements was applied to treat the processes involving fluid-solid interfaces, both the shear stress and the mean separation profiles were in good agreement with the fully atomistic results [26].

The present paper first examines the effects of the coarsegraining procedure with local elements in the dynamically constrained and free-energy-corrected HACG simulations on the transverse stress of the thin-film lubrication system. Second, the influences of nonlocal elements in the freeenergy-corrected HACG simulation on the thermomechnical properties are determined. Third, when the macroscopic thermomechanical quantities in the fully atomistic simulation can be successively predicted by using the free-energy-corrected HACG method, the deviation of the atomistic positions of the solid substrates in the coarse-graining description from those in the fully atomistic description is further investigated.

\section{TRIBOLOGICAL SYSTEM AND FREE-ENERGY-CORRECTED HACG METHOD}

Figure 1(a) is a schematic diagram of the idealized 2D contact, which consists of two identical crystalline substrates separated by a thin fluid film. The top and bottom walls are assumed to be rigid with the nearest-neighbor distance fixed as the lattice constant $a$ of the hexagonal close-packed structure. The substrates are connected to the top/bottom walls and divided into far and near regions, where the solid atoms with the initial hexagonal close-packed structure are placed. In Figs. 1(b), 1(c), the far region of each substrate is coarsely divided with a finite-element mesh and connected to the near region of the substrate. The system is bounded by an $L_{x} \times L_{y}$ rectangle with a periodic boundary condition in the $x$ direction. The sliding distance of the top wall is specified by the registry $\alpha$, defined as

$$
x_{i}^{t}=x_{i}^{b}+\alpha a,
$$

where $t$ and $b$, respectively, denote $x$ coordinates of the corresponding atoms in the top and bottom walls and $\alpha$ is the distance in the unit of the lattice constant by which the top wall is displaced laterally with respect to the bottom wall. In the coarse-grained portion of each substrate, the nodes of congruent equilateral triangles in the finite-element mesh coincide with a subset of solid and wall atoms. The coordinates $r_{i \alpha}$ of the underlying atom $i$ can be written in terms of the nodal coordinates $\left(R_{k \alpha}\right)$ as

$$
r_{i \alpha}=\sum_{k=1}^{3} A_{i}(k) R_{k \alpha} / A_{e} ; \alpha=x, y,
$$

where $k$ is the index of the nodes, $A_{e}$ denotes the area of an element $e$ and $A_{i}(k)$ denotes the area of the inscribed triangle with one side coinciding with the side of the element $e$ opposite node $k$ and the opposite vertex coinciding with the lattice site at $\left(r_{i x}, r_{i y}\right)$.

The coarse-graining procedure partitions the original solid atoms into two subsets: $N_{n}$ is the number of the nodal atoms and $N_{s}$ is the number of the non-nodal atoms. As shown in Fig. 1(b), all elements are local, the contribution of the coarse-grained positions of the substrates to the effective configurational energy can thus be expressed as

$$
V_{c f}\left(\mathbf{R}^{N_{n}}\right)=\sum_{e=1}^{N_{e}}\left(N_{a}^{e} \tilde{u}_{e}+N_{s}^{e} f_{e}\right)
$$

where $\mathbf{R}^{N_{n}}$ stands for the nodal configuration, $N_{e}$ for the number of elements, $N_{a}^{e}$ the number of atoms underlying the element $e, N_{s}^{e}$ the number of non-nodal atoms underlying the element $e$. The configurational energy per atom $\tilde{u}_{e}$ is expressed as

$$
\tilde{u}_{e}=\frac{1}{2} \sum_{j \neq i} u_{s s}\left(r_{i j}\right)
$$

where $i$ denotes the centroid atom (i.e., the atom nearest the centroid of $e$ ) and $j$ is the index of the atoms that lie within the circle of radius $r_{c}$ centered on $i$. We take account of the pair interactions by the shifted Lennard-Jones $(12,6)$ potentials

$$
u_{a b}(r)= \begin{cases}\phi_{a b}(r)-\phi_{a b}\left(r_{c}\right), & \text { if } r<r_{c} ; \\ 0, & \text { if } r \geqslant r_{c},\end{cases}
$$

where $r_{c}$ is the cut-off radius and

$$
\phi_{a b}(r)=4 \epsilon_{a b}\left[(\sigma / r)^{12}-(\sigma / r)^{6}\right], \quad a b=f f, f s, s s .
$$

The effective diameter $\sigma, \epsilon_{a b}$ and the range $r_{c}$ are the same for all pairs. By using the local harmonic approximation [27], the 
Helmholtz energy per atom $f_{e}$ can be estimated as

$$
f_{e}=2 k_{B} T \ln \left[h(\operatorname{det} D)^{1 / 4} / k_{B} T\right],
$$

where the elements of the $2 \times 2$ dynamical matrix are $D_{k l}=m^{-1}\left(\partial^{2} \tilde{u}_{e} / \partial x_{k} \partial x_{l}\right)_{0}(k, l=1,2), k$ and $l$ are indices of Cartesian components $\left(x_{1}=x, x_{2}=y\right)$ of the position of the reference atom, and the subscript 0 signifies that the partial derivative is evaluated at the equilibrium configuration. It is noted that when the last term in Eq. (3) is removed, $V_{c f}$ will be reduced to the coarse-grained configurational energy under the dynamical constraint.

Figure 1(c) displays the finite-element meshes with nonlocal elements in the coarse-grained solid substrates. In the merging regions between the atomistic description in the near region and the finite-element mesh in the far regions of the solid substrates, the solid atoms in the near region are not always in an equilibrium configuration of the elements, but have an inhomogeneous distortion. In the free-energy-corrected HACG simulations, the coarse-graining treatment with respect to the local elements reduces the effects of the inhomogeneous distortion of the solid atoms in the near regions of the substrates on the element energy. However, in the coarse-graining treatment with respect to the nonlocal elements, the element energy includes not only the interactions of the solid atoms in itself and in its neighboring elements, but also the effects of the solid atoms in the near regions. In the merging regions, the cut-off circles of the nonlocal elements cover some solid atoms in the near regions. The inhomogeneous solid atoms replace the homogeneous ones around the reference atoms at the equilibrium configuration in Eq. (7). The free-energycorrected HACG simulation for the nonlocal elements shows that the solid atoms in the near regions strongly affect the interaction between the near atomistic regions and the far coarse-graining regions. It leads to an unphysical sliding at the interface between them of the upper substrate. For example, the forward shearing process of the tribological system at temperature $T=0.05$ simulated by the free-energy-corrected HACG method for the nonlocal elements is shown in Fig. 2 and compared with the results in the fully atomistic simulation. The unphysical sliding appears in the upper interface between the near atomistic region and far coarse-graining region at $\alpha=$ 1.00 and replaces the physical sliding in the thin film between two near regions at $\alpha=0.50$. Therefore, in the merging regions, we remove the free-energy correction and keep the dynamical constraint for the nonlocal elements connected with the solid atoms. In this case, Eq. (3) is rewritten as

$$
V_{c f}\left(\mathbf{R}^{N_{n}}\right)=\sum_{e=1}^{N_{e}} N_{a}^{e} \tilde{u}_{e}+\sum_{e=1}^{N_{e}^{\prime}} N_{s}^{e} f_{e},
$$

(a)
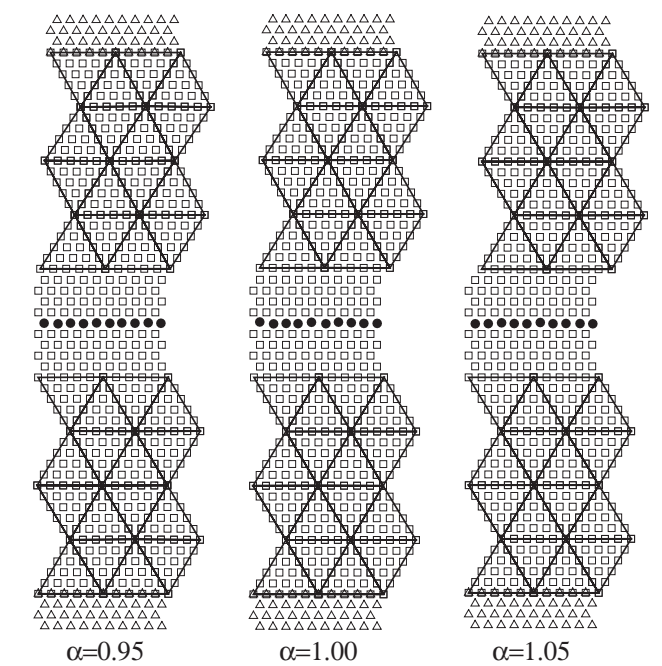

(b)

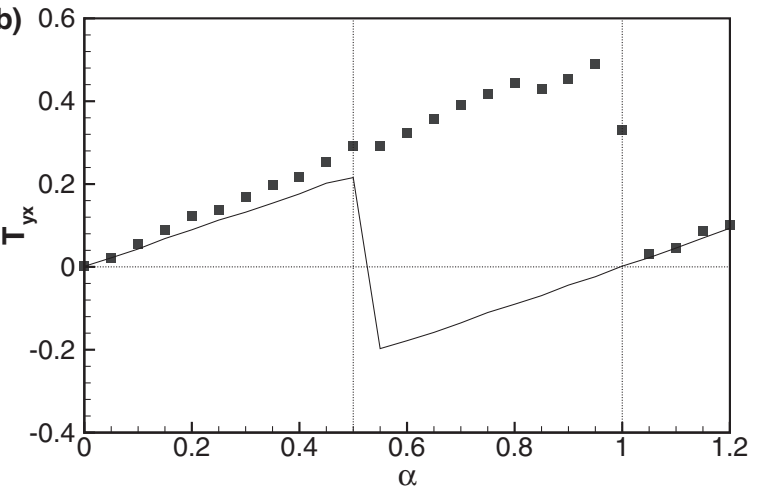

FIG. 2. Forward shearing process of the tribological system at temperature $T=0.05$ simulated by the free-energy-corrected HACG method for the nonlocal elements. (a) snapshot diagrams at the quasistatic states $\alpha=0.95,1.00$, and 1.05; (b) shear-stress profiles ( $T_{y x}$ vs register $\alpha$ ) for fully atomistic profile $T_{y x}(\alpha)$ (solid line) and free-energy-corrected HACG $T_{y x, c f}(\alpha)$ (dark gray filled squares).

where $N_{e}^{\prime}$ stands for the number of elements, which are not in the merging regions. In Eq. (8), the free-energy contribution of the non-nodal atoms in the elements $\left(N_{e}-N_{e}^{\prime}=8\right)$ of the merging regions is reduced to prevent the unphysical sliding between the near and far regions of the upper substrate. In what follows, the thermomechanical quantities of the tribological system are always derived with respect to the local elements, the similar derivations of the thermomechanical properties with respect to the nonlocal elements are omitted for convenience.

The total configurational energy of the free-energycorrected HACG system is then given by

$$
\begin{aligned}
U_{c f}\left(\mathbf{r}^{N_{f}}, \mathbf{r}^{N_{s}^{\prime}}, \mathbf{R}^{N_{n}}\right)= & \frac{1}{2} \sum_{i=1}^{N_{f}} \sum_{j \neq i}^{N_{f}} u_{f f}\left(r_{i j}\right)+\sum_{i=1}^{N_{f}} \sum_{j=1}^{N_{s}^{\prime}} u_{f s}\left(r_{i j}\right)+\frac{1}{2} \sum_{i=1}^{N_{s}^{\prime}} \sum_{j \neq i}^{N_{s}^{\prime}} u_{s s}\left(r_{i j}\right)+\frac{1}{2} \sum_{i=1}^{N_{s}^{\prime}} \sum_{j=1}^{N_{s}^{\prime \prime}} u_{s s}\left(r_{i j}\right) \\
& +\frac{1}{2} \sum_{i=1}^{N_{w}} \sum_{j=1}^{N_{s}^{\prime \prime}} u_{s s}\left(r_{i j}\right)+\sum_{e=1}^{N_{e}} N_{a}^{e}\left[\frac{1}{2} \sum_{j \neq i} u_{s s}\left(r_{i j}\right)\right]+\sum_{e=1}^{N_{e}} N_{s}^{e} 2 k_{B} T \ln \left[h(\operatorname{det} D)^{1 / 4} / k_{B} T\right],
\end{aligned}
$$


where

$$
\begin{aligned}
& |D|=D_{x x} D_{y y}-D_{x y}^{2}, \\
& D_{x x}=m^{-1} \sum_{j \neq i}\left\{u_{s s}^{\prime \prime} x_{i j}^{2} / r_{i j}^{2}+u_{s s}^{\prime} y_{i j}^{2} / r_{i j}^{3}\right\} \\
& D_{y y}=m^{-1} \sum_{j \neq i}\left\{u_{s s}^{\prime \prime} y_{i j}^{2} / r_{i j}^{2}+u_{s s}^{\prime} x_{i j}^{2} / r_{i j}^{3}\right\} \\
& D_{x y}=m^{-1} \sum_{j \neq i}\left\{u_{s s}^{\prime \prime} x_{i j} y_{i j} / r_{i j}^{2}-u_{s s}^{\prime} x_{i j} y_{i j} / r_{i j}^{3}\right\}
\end{aligned}
$$

and $N_{s}^{\prime}$ stands for the number of atoms in the near regions of the substrate (not coarse-grained), $N_{s}^{\prime \prime}$ for the number of underlying (coarse-grained) atoms, and $N_{w}$ is the number of wall atoms. The shear stress $T_{y x, c f}$ and the transverse stress $T_{x x, c f}$ of the free-energy-corrected HACG system are, respectively, given by

$$
\begin{aligned}
T_{y x, c f}= & \frac{1}{2 L_{x}} \sum_{i=1}^{N_{f}} \sum_{j \neq i}^{N_{f}}\left\langle u_{f f}^{\prime}\left(r_{i j}\right) x_{i j} y_{i j} /\left(r_{i j} L_{y}\right)\right\rangle+\frac{1}{L_{x}} \sum_{i=1}^{N_{f}} \sum_{j=1}^{N_{s}^{\prime}}\left\langle u_{f s}^{\prime}\left(r_{i j}\right) x_{i j} y_{i j} /\left(r_{i j} L_{y}\right)\right\rangle+\frac{1}{2 L_{x}} \sum_{i=1}^{N_{s}^{\prime}} \sum_{j \neq i}^{N_{s}^{\prime}}\left\langle u_{s s}^{\prime}\left(r_{i j}\right) x_{i j} y_{i j} /\left(r_{i j} L_{y}\right)\right\rangle \\
& +\frac{1}{2 L_{x}} \sum_{i=1}^{N_{s}^{\prime}} \sum_{j=1}^{N_{s}^{\prime \prime}}\left\langle u_{s s}^{\prime}\left(r_{i j}\right) x_{i j} y_{i j} /\left(r_{i j} L_{y}\right)\right\rangle+\frac{1}{2 L_{x}} \sum_{i=1}^{N_{w}} \sum_{j=1}^{N_{s}^{\prime \prime}}\left\langle u_{s s}^{\prime}\left(r_{i j}\right) x_{i j} y_{i j} /\left(r_{i j} L_{y}\right)\right\rangle \\
& +\frac{1}{L_{x}} \sum_{e=1}^{N_{e}} N_{a}^{e}\left[\frac{1}{2} \sum_{j \neq i}\left\langle u_{s s}^{\prime}\left(r_{i j}\right) x_{i j} y_{i j} /\left(r_{i j} L_{y}\right)\right\rangle\right]+\frac{1}{L_{x}} \sum_{e=1}^{N_{e}} N_{s}^{e}\left[\frac{k_{B} T}{2}\left\langle\frac{1}{|D|} \frac{\partial|D|}{\partial \alpha a}\right\rangle\right]
\end{aligned}
$$

and

$$
\begin{aligned}
T_{x x, c f}= & -\frac{\left(N_{f}+N_{s}^{\prime}+N_{n}\right) k_{B} T}{L_{x}\left\langle L_{y}\right\rangle}+\frac{1}{2 L_{x}} \sum_{i=1}^{N_{f}} \sum_{j \neq i}^{N_{f}}\left\langle u_{f f}^{\prime}\left(r_{i j}\right) x_{i j}^{2} /\left(r_{i j} L_{y}\right)\right\rangle+\frac{1}{L_{x}} \sum_{i=1}^{N_{f}} \sum_{j=1}^{N_{s}^{\prime}}\left\langle u_{f s}^{\prime}\left(r_{i j}\right) x_{i j}^{2} /\left(r_{i j} L_{y}\right)\right\rangle \\
& +\frac{1}{2 L_{x}} \sum_{i=1}^{N_{s}^{\prime}} \sum_{j \neq i}^{N_{s}^{\prime}}\left\langle u_{s s}^{\prime}\left(r_{i j}\right) x_{i j}^{2} /\left(r_{i j} L_{y}\right)\right\rangle+\frac{1}{2 L_{x}} \sum_{i=1}^{N_{s}^{\prime}} \sum_{j=1}^{N_{s}^{\prime \prime}}\left\langle u_{s s}^{\prime}\left(r_{i j}\right) x_{i j}^{2} /\left(r_{i j} L_{y}\right)\right\rangle+\frac{1}{2 L_{x}} \sum_{i=1}^{N_{w}} \sum_{j=1}^{N_{s}^{\prime \prime}}\left\langle u_{s s}^{\prime}\left(r_{i j}\right) x_{i j}^{2} /\left(r_{i j} L_{y}\right)\right\rangle \\
& +\frac{1}{L_{x}} \sum_{e=1}^{N_{e}} N_{a}^{e}\left[\frac{1}{2} \sum_{j \neq i}\left\langle u_{s s}^{\prime}\left(r_{i j}\right) x_{i j}^{2} /\left(r_{i j} L_{y}\right)\right\rangle\right]+\frac{1}{L_{x}} \sum_{e=1}^{N_{e}} N_{s}^{e}\left[\frac{k_{B} T}{2} \sum_{l}\left\langle\frac{1}{|D|} \frac{\partial|D|}{\partial r_{l \alpha}} r_{l \alpha}\right\rangle\right] .
\end{aligned}
$$

\section{RESULTS}

We consider the behavior of the system under a reversible shearing with a constant number of atoms, a constant temperature, and a fixed load $-T_{y y}=0.1$, To compute the thermomechanical properties we carry out the isothermalisobaric Monte Carlo (MC) simulations using the procedures given previously [24,28,29]. Table I lists the values of the various parameters for the simulations. Numerical values are expressed in dimensionless units based on the LennardJones (argon) parameters for the solid-solid interaction: the distance is expressed in the unit of $\sigma$; the energy in the unit of $\epsilon_{s s}$; the stress in the unit of $\epsilon_{s s} / \sigma$; the temperature in the unit of $\epsilon_{s s} / k_{B}$. We use the local and nonlocal elements in the coarse-graining procedures for substrates to investigate the effects of the coarse graining. The computational details of thermomechanical properties of the system by the free-energy-corrected QC method with the coarsegraining local (nonlocal) elements are summarized in the appendix.

\section{A. Thermomechanical properties}

\section{Local elements in the coarse-grained substrates}

Figure 3(a) shows the shear stress $T_{y x, c f}$ at temperature $T=$ 0.1 versus register $\alpha$. The curves corresponding to shearing from $\alpha=0$ to $\alpha=1.2$ and to reversing from $\alpha=1.2$ to $\alpha=0$. The profile $T_{y x, c f}(\alpha)$ is periodic in the range $0 \leqslant \alpha<1.0$ and antisymmetric about $\alpha=0.5$. In the forward direction, as $\alpha$ varies in the range $0 \leqslant \alpha<0.4$, the tribological system undergoes a slip process, where the friction force increases with the gradual shearing. Then, in the range $0.4<\alpha<0.6$, the friction force reaches the maximum and the tribological system undergoes a stick process. Finally, as $\alpha$ varies in the ranges $0.6<\alpha<1.0$, the tribological system undergoes a reversed slip process and returns to its end state $\alpha=1.0$, i.e., the initial state $\alpha=0$. In the backward direction, the reversing from $\alpha=1.0$ to $\alpha=0$ carries the tribological system through the same states as the shearing from $\alpha=0$ to $\alpha=1.0$. Figure 3(a) compares the results of the exact $T_{y x}$ and the dynamically constrained HACG $T_{y x, c}$. The curves of 
TABLE I. Parameters of the multiscale simulation.

Number of film atoms $N_{f}=10$

Number of wall atoms $N_{w}=2 \times 40=80$

Total number of substrate atoms $N_{s}=2 \times 240=480$

Number of near-region atoms $N_{s}^{\prime}=2 \times 40=80$

Number of far-region atoms $N_{s}^{\prime \prime}=2 \times 200=400$

Number of local/nonlocal elements $N_{e}=2 \times 4 / 16=8 / 32$

Number of nodes in local/nonlocal elements $N_{n}=2 \times 2 / 8=4 / 16$

Lattice constant of substrate $a=\sqrt{\frac{2}{\sqrt{3}}}=1.075$

Width of contact $L_{x}=10 a$

Substrate-substrate Lennard-Jones depth $\epsilon_{s s}=1$

Film-film Lennard-Jones depth $\epsilon_{f f}=1 / 9$

Substrate-film Lennard-Jones depth $\epsilon_{s f}=1 / 9$

Cutoff radius $r_{c}=2.5$

Total number of Monte Carlo cycles $M=10^{6}$

Mass of argon atom $m_{s}=6.63 \times 10^{-27} \mathrm{~kg}$

Length scale of argon $\sigma=3.41 \times 10^{-10} \mathrm{~m}$

Well depth of $\operatorname{argon} \epsilon_{s}=1.66 \times 10^{-21} \mathrm{~J}$

the free-energy-corrected HACG, the dynamically constrained HACG and the exact shear stress are in excellent agreement, which shows that the both HACG methods can reliably be used to approximate the exact shear stress response of the system. Figure 3(b) shows the mean separation distance $\left\langle L_{y, c f}\right\rangle$ between the top and bottom walls versus $\alpha$. The profile $\left\langle L_{y, c f}\right\rangle(\alpha)$ is also periodic in the range $0 \leqslant \alpha<1.0$ but symmetric about $\alpha=0.5$. The free-energy-corrected HACG $\left\langle L_{y, c f}\right\rangle(\alpha)$ and the exact $\left\langle L_{y}\right\rangle(\alpha)$ are in excellent agreement, but the dynamically constrained HACG $\left\langle L_{y, c}\right\rangle(\alpha)$ is shorter than the exact $\left\langle L_{y}\right\rangle(\alpha)$. The difference is due to the fact that the coarse-grained portion of the lattice can undergo only a restricted thermal expansion on account of the dynamical constraints on the underlying atoms in the dynamically constrained HACG simulation. However, the free-energy-corrected HACG method recovers the above missing thermal expansions of the non-nodal atoms in the elements. Figure 3(c) shows the transverse stress $T_{x x, c f}$ versus $\alpha$. The profile $T_{x x, c f}(\alpha)$ is also periodic in the range $0 \leqslant \alpha<1.0$ and symmetric about $\alpha=0.5$. The free-energy-corrected HACG $T_{x x, c f}$ is a little larger than the exact $T_{x x}$, but the dynamically constrained HACG $T_{x x, c}$ is markedly less than the exact $T_{x x}$. In Table II, it is seen that the deviation of $T_{x x, c f}$ from $T_{x x}$ at $\alpha=0$ is only $2.1 \%$, but the deviation of $T_{x x, c}$ from $T_{x x}$ at $\alpha=0$ is as large as $10.0 \%$. Table II also lists the contributions of the ideal-gas interaction $\tau_{\mathrm{IG}}$ and the thermally averaged interaction $\tau_{e x}$ in the transverse stress $T_{x x}$. In comparison with the exact $\tau_{\mathrm{IG}}$ and $\tau_{e x}$, it is found that the deviation of $T_{x x, c f}$ is mainly resulted from the missing contribution of the ideal-gas interaction, and the deviation of $T_{x x, c}$ is mainly resulted from the missing contribution of the thermally averaged interaction.

In order to investigate the effects of temperature on the freeenergy-corrected HACG method, we simulate the tribological process at temperature $T=0.05$ and the results are shown in Fig. 4. As $\alpha$ varies over the range $0 \leqslant \alpha<1.0$, the tribological system undergoes a similar process observed above at $T=0.1$, except that a delay stick process happens in the range $0.5<$ $\alpha<0.6 / 0.4<\alpha<0.5$ in the forward/backward directions. $T_{y x, c f}$ and $\left\langle L_{y, c f}\right\rangle$ generated by the free-energy-corrected

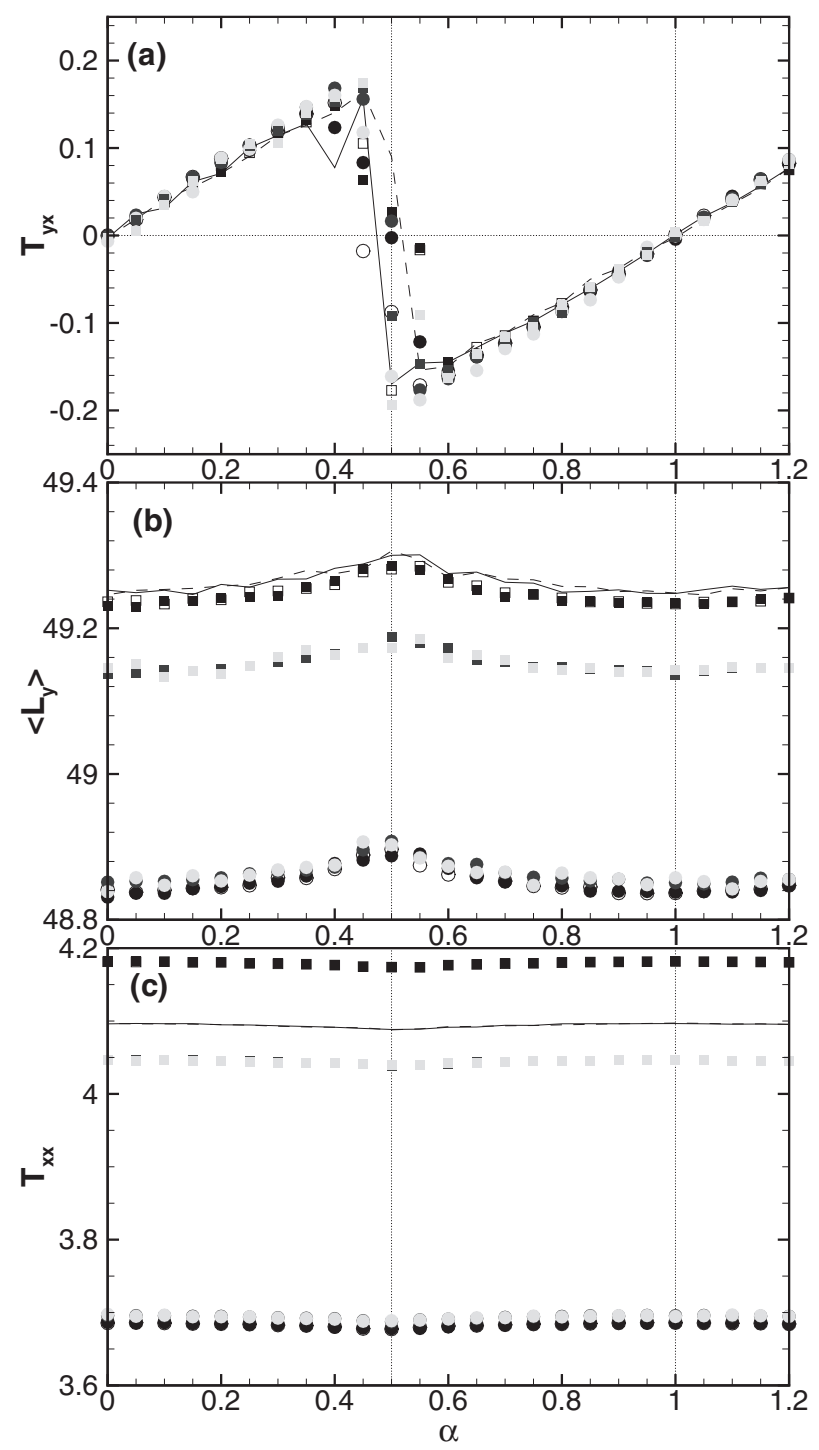

FIG. 3. (a) Shear-stress profile $\left(T_{y x}\right.$ vs register $\left.\alpha\right)$ (b) mean separation between walls $\left\langle L_{y}\right\rangle$ vs register $\alpha$ (c) transverse-stress profile ( $T_{x x}$ vs register $\alpha$ ) for model contact with monolayer film at temperature $T=0.1$. Local or nonlocal elements are placed in the coarse-grained substrates. Fully atomistic profile $T_{y x}(\alpha)$ (forward, by solid line; reverse, by dashed line); dynamically constrained HACG $T_{y x, c}(\alpha)$ calculated with the local or nonlocal elements (forward, by black/dark gray filled circles; reverse, by white/light gray filled circles); free-energy-corrected HACG $T_{y x, c f}(\alpha)$ calculated with the local or nonlocal elements (forward, by black/dark gray filled squares; reverse, by white/light gray filled squares).

HACG simulations are in excellent agreement with the results of the fully atomistic simulations. Although the dynamically constrained HACG $T_{y x, c}(\alpha)$ is the same as the exact $T_{y x}(\alpha)$, $\left\langle L_{y, c}\right\rangle(\alpha)$ is shorter than the exact $\left\langle L_{y}\right\rangle(\alpha)$. The free-energycorrected HACG $T_{x x, c f}$ is a little larger than the exact $T_{x x}$, but the dynamically constrained HACG $T_{x x, c}$ is markedly less than the exact $T_{x x}$. In Table II, at temperature $T=0.05$, the deviations of $T_{x c, c f}$ and $T_{x x, c}$ from $T_{x x}$ at $\alpha=0$ are less than those at $T=0.1$ and are only $1.0 \%$ and $5.4 \%$. Therefore, the increase (decrease) of temperature intensifies (abates) the 
TABLE II. Transverse stress $\left(T_{x x}\right)$ and contributions of ideal-gas and thermally averaged interactions $\left(T_{x x}=\tau_{\mathrm{IG}}+\tau_{e x}\right)$ for the fully atomistic, dynamically constrained HACG and free-energy-corrected HACG simulations as a function of temperature ( $T$ ) for two-dimensional tribological system at register $\alpha=0$. Number in parentheses after entry is absolute percent error based on atomistic value.

\begin{tabular}{|c|c|c|c|c|c|}
\hline$T$ & $\begin{array}{c}\text { Atomistic } \\
T_{x x}\end{array}$ & $\begin{array}{l}\text { Local Ele. } \\
\qquad T_{x x, c}\end{array}$ & $\begin{array}{c}\left(N_{n}=4\right) \\
T_{x x, c f}\end{array}$ & $\begin{array}{l}\text { Nonlocal Ele. } \\
\qquad T_{x x, c}\end{array}$ & $\begin{array}{c}\left(N_{n}=16\right) \\
T_{x x, c f}\end{array}$ \\
\hline 0.05 & 3.859 & $3.649(5.4)$ & $3.897(1.0)$ & $3.654(5.3)$ & $3.830(0.8)$ \\
\hline 0.1 & 4.096 & $3.686(10.0)$ & $4.182(2.1)$ & $3.696(9.8)$ & $4.047(1.2)$ \\
\hline$T$ & $\tau_{\mathrm{IG}} / \tau_{e x}$ & $\tau_{\mathrm{IG}, c} / \tau_{e x, c}$ & $\tau_{\mathrm{IG}, c f} / \tau_{e x, c f}$ & $\tau_{\mathrm{IG}, c} / \tau_{e x, c}$ & $\tau_{\mathrm{IG}, c f} / \tau_{e x, c f}$ \\
\hline$\overline{0.05}$ & $-0.047 / 3.906$ & $-0.009 / 3.658$ & $-0.009 / 3.906$ & $-0.010 / 3.664$ & $-0.010 / 3.840$ \\
\hline 0.1 & $-0.093 / 4.189$ & $-0.018 / 3.704$ & $-0.018 / 4.200$ & $-0.020 / 3.716$ & $-0.020 / 4.067$ \\
\hline
\end{tabular}

restriction of the thermal expansion of the non-nodal atoms in the elements for both $\tau_{\mathrm{IG}}$ and $\tau_{\text {ex }}$.

The comparisons show that the current multiscale method, i.e., the HACG with the free-energy correction for the nonnodal atoms in the local elements can successfully reduce the effects of the dynamical constraint in the coarse-graining

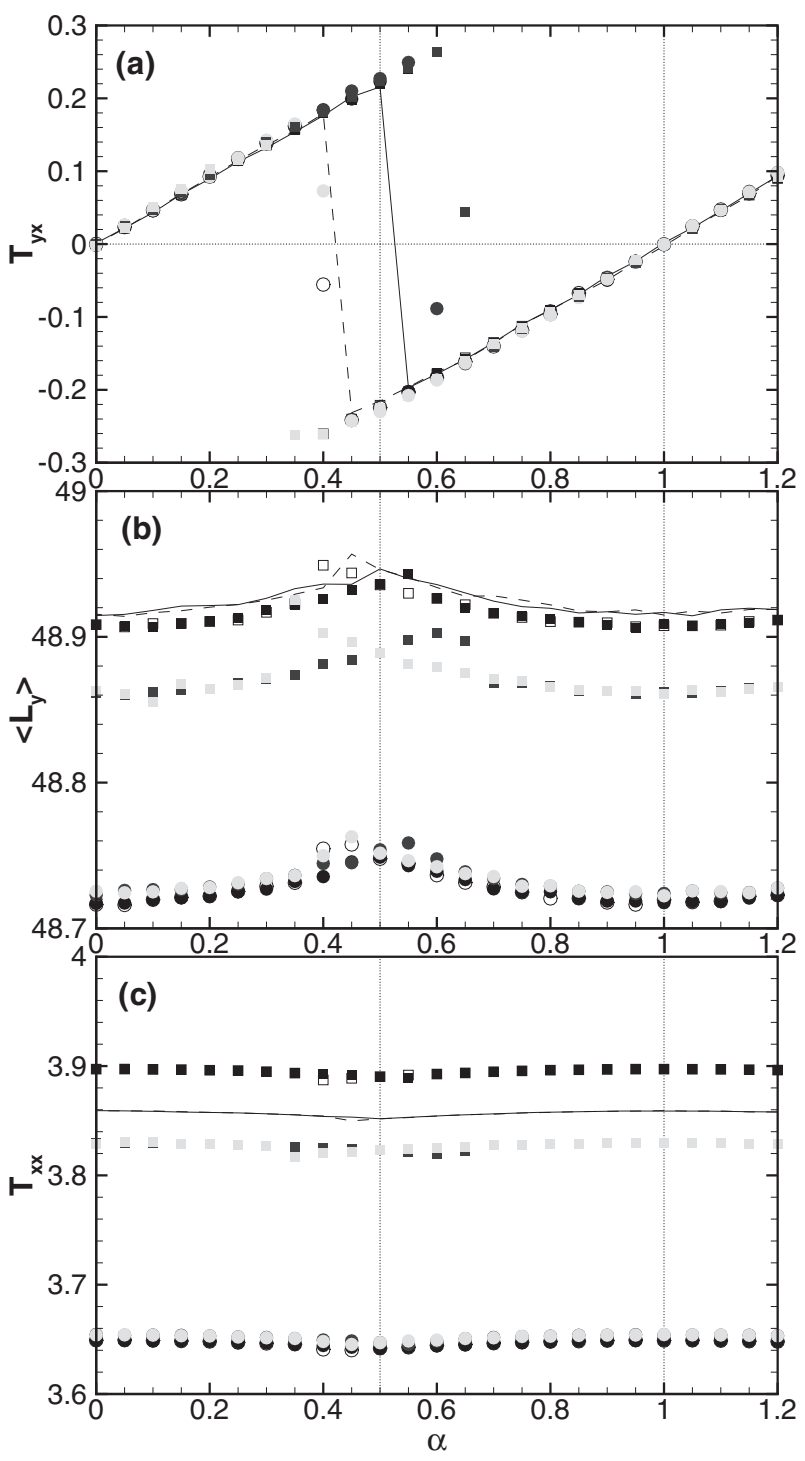

FIG. 4. Same as Fig. 3, except $T=0.05$. procedure. It can be concluded that the multiscale simulation results agree very well with the fully atomistic ones for the tribological system with respect to both the shear stress and the mean separation profiles. It can also provide a better approximation to the transverse stress of the tribological system than the dynamically constrained HACG method.

\section{Nonlocal elements in the coarse-grained substrates}

Figure 3(a) shows the shear stresses $T_{y x, c f}, T_{y x}$, and $T_{y x, c}$ at temperature $T=0.1$ versus register $\alpha$. The curves of the free-energy-corrected HACG, the dynamically constrained HACG, and the exact shear stress are in excellent agreement. Figure 3(b) shows the mean separation distance $\left\langle L_{y, c f}\right\rangle,\left\langle L_{y}\right\rangle$ and $\left\langle L_{y, c}\right\rangle$ between the top and bottom walls versus $\alpha$. The mean separation curve $\left\langle L_{y, c f}\right\rangle$ obtained by the free-energycorrected HACG simulation is a little shorter than the exact $\left\langle L_{y}\right\rangle$, but $\left\langle L_{y, c}\right\rangle$ obtained by the dynamically constrained HACG simulation is markedly shorter than the exact $\left\langle L_{y}\right\rangle$. Figure 3(c) shows the transverse stresses $T_{x x, c f}, T_{x x}$, and $T_{x x, c}$ versus $\alpha . T_{x x, c f}$ from the free-energy-corrected HACG simulation is a little smaller than the exact $T_{x x}$, but $T_{x x, c}$ from the dynamically constrained HACG simulation is markedly smaller than the exact $T_{x x}$. Table II shows that the deviation of $T_{x x, c f}$ from $T_{x x}$ at $\alpha=0$ is only $1.2 \%$, but the deviation of $T_{x x, c}$ from $T_{x x}$ at $\alpha=0$ is as large as 9.8\%. In comparison with the exact $\tau_{\mathrm{IG}}$ and $\tau_{e x}$, it is found that the deviation of $T_{x x, c f}$ is mainly resulted from the missing contribution of the ideal-gas interaction, on the other hand, the deviation of $T_{x x, c}$ is mainly resulted from the missing contribution of the thermally averaged interaction.

Figure 4 displays the tribological process at temperature $T=0.05$ simulated by using the free-energy-corrected HACG method. In comparison with the fully atomistic and the dynamically constrained HACG simulations, $T_{y x, c f},\left\langle L_{y, c f}\right\rangle$, and $T_{x x, c f}$ generated by the free-energy-corrected HACG method are similar to those at $T=0.1$, except that their errors are reduced for $\left\langle L_{y, c f}\right\rangle$ and $T_{x x, c f}$ and increased for $T_{y x, c f}$ in the snapping processes as the temperature decreases. In Table II, at $T=0.05$, the deviations of $T_{x x, c f}$ and $T_{x x, c}$ from $T_{x x}$ at $\alpha=0$ are only $0.8 \%$ and $5.3 \%$. Therefore, the increase (decrease) of temperature intensifies (abates) the restriction of the thermal expansion of the non-nodal atoms in the elements for both $\tau_{\mathrm{IG}}$ and $\tau_{\text {ex }}$.

The comparisons show that the current multiscale method, i.e., the HACG with a free-energy correction for the non-nodal atoms in the nonlocal elements can successfully reduce the 
effects of the dynamical constraint in the coarse-graining procedure. It can thus be concluded that the multiscale simulation results are agree very well with the fully atomistic results for the tribological system with respect to the shear stress profile. It can also provide a better approximation to both the mean separation and the transverse stress profiles for the tribological system than the dynamically constrained HACG method.

\section{B. Deviations of atomistic positions in coarse-grained substrates}

\section{Local elements in the coarse-grained substrates}

Although the macroscopic thermomechanical quantities in the fully atomistic simulations can be successively predicted by using the free-energy-corrected HACG method, it is important to know the deviations of the atomistic equilibrium positions of the substrates in the coarse-graining description from those in the fully atomistic description. Consider the tribological system at $\alpha=0$ to investigate the deviations of the atomistic positions in the coarse-grained substrates from those in the fully atomistic substrates.

When the system is in equilibrium states, each atom vibrates near its equilibrium position and has different instantaneous positions $\left(x_{i}^{s}, y_{i}^{s}\right)$. Its equilibrium position $\left(\bar{x}_{i}, \bar{y}_{i}\right)$ can be determined by an average of $M_{s}$ different instantaneous positions and defined as follows

$$
\bar{x}_{i}=\frac{1}{M_{s}} \sum_{s=1}^{M_{s}} x_{i}^{s}, \quad \bar{y}_{i}=\frac{1}{M_{s}} \sum_{s=1}^{M_{s}} y_{i}^{s} .
$$

Then, the fluctuation of the atomistic positions of the substrates in the fully atomistic description and the mean distance between the atomistic positions of the substrates in the coarsegraining description and those in the fully atomistic description are defined, respectively, as

$$
\begin{aligned}
r_{i} & =\sqrt{\frac{1}{M_{s}} \sum_{s=1}^{M_{s}}\left[\left(x_{i}^{s}-\bar{x}_{i}\right)^{2}+\left(y_{i}^{s}-\bar{y}_{i}\right)^{2}\right]}, \\
\bar{r} & =\frac{1}{N} \sum_{i=1}^{N} r_{i}
\end{aligned}
$$

and

$$
d_{c / c f}=\sqrt{\frac{1}{N} \sum_{i=1}^{N}\left[\left(\bar{x}_{i, c / c f}-\bar{x}_{i}\right)^{2}+\left(\bar{y}_{i, c / c f}-\bar{y}_{i}\right)^{2}\right]} .
$$

In the fully atomistic, the dynamically constrained HACG and the free-energy-corrected HACG simulations, we take snapshots of the system states at MC cycles of $M=9.0 \times 10^{5}$, $9.5 \times 10^{5}, 1.0 \times 10^{6}, 1.05 \times 10^{6}$, and $1.1 \times 10^{6}$ with $M_{s}=5$ for different instantaneous positions in the equilibrium states of the system. In the snapshots for the dynamically constrained HACG and the free-energy-corrected HACG simulations, the non-nodal atomistic positions in the elements are directly determined from the nodal atomistic coordinates. Using Eqs. (14) and (15), we calculate $\bar{r}$ and $d_{c}$, as listed in Table III. It is evident that $d_{c}$ is far away from $\bar{r}$, but $d_{c f}$ is close to $\bar{r}$ at $T=0.1$. This means that the atomistic equilibrium positions of the substrates in the free-energy-corrected HACG
TABLE III. Fluctuation of atomistic positions of substrates in the fully atomistic description and mean deviations of atomistic positions in the both coarse-grained substrates from those in the fully atomistic substrates as a function of temperature $(T)$ for two-dimensional tribological system at register $\alpha=0$.

\begin{tabular}{lccccc}
\hline \hline & $\begin{array}{c}\text { Atomistic } \\
T\end{array}$ & $\begin{array}{c}\text { Local Ele. } \\
d_{c}\end{array}$ & $\begin{array}{c}\left(N_{n}=4\right) \\
d_{c f}\end{array}$ & $\begin{array}{c}\text { Nonlocal Ele. } \\
d_{c}\end{array}$ & $\begin{array}{c}\left(N_{n}=16\right) \\
d_{c f}\end{array}$ \\
\hline 0.05 & 0.047 & 0.052 & 0.029 & 0.056 & 0.055 \\
0.1 & 0.072 & 0.117 & 0.049 & 0.105 & 0.056 \\
\hline \hline
\end{tabular}

simulation are closer to those in the fully atomistic simulation than those in the dynamically constrained HACG simulation. However, both $d_{c}$ and $d_{c f}$ are close to $\bar{r}$ at $T=0.05$. This means that the fluctuations of the atomistic positions of the substrates in the fully atomistic, the dynamically constrained HACG and the free-energy-corrected HACG descriptions are reduced as $T$ decreases. The atomistic equilibrium positions of the substrates in both the free-energy-corrected and the dynamically constrained HACG simulations are approximately placed with respect to the fluctuation of the atomistic positions of the substrates in the fully atomistic simulation. Therefore, the free-energy-corrected HACG simulation not only can predict the consistent macroscopic thermomechanical quantities observed in the fully atomistic simulation, but also can predict the microscopic atomistic positions with respect to the fluctuation of those in the fully atomistic simulation.

\section{Nonlocal elements in the coarse-grained substrates}

For the coarse-grained substrates with nonlocal elements, using the above same method, we calculate $\bar{r}$ and $d_{c / c f}$, as listed in Table III. It is evident that $d_{c}$ is far different from $\bar{r}$, but $d_{c f}$ is close to $\bar{r}$ at $T=0.1$. However, $d_{c}$ and $d_{c f}$ are close to $\bar{r}$ at $T=0.05$. Therefore, the atomistic equilibrium positions of the substrates in the free-energy-corrected HACG simulation are closer to those in the fully atomistic simulation than those in the dynamically constrained HACG simulation. As $T$ decreases, the atomistic equilibrium positions of the substrates in both the free-energy-corrected and dynamically constrained HACG simulations are approximately placed with respect to the fluctuation of the atomistic positions of the substrates in the fully atomistic simulation.

\section{CONCLUSION}

In this paper, the free-energy-corrected QC method at nonzero temperature is applied for a thin-film lubrication system. The local and nonlocal elements are placed in the coarse-grained substrates, respectively. It is shown that the multiscale method with the coarse-graining local elements in the merging regions between the atomistic and continuous descriptions of the substrates can reasonably predict the shear stress profile, the mean separation curve, and the transverse stress profile in the fully atomistic simulation for the tribological system. Moreover, when the nonlocal elements are placed in the merging regions, the inhomogeneous solid atoms in the near regions covered by the cut-off circles of the nonlocal elements replace the homogeneous ones at the equilibrium configuration for the free-energy correction to 
the non-nodal atoms. The treatment results in an unphysical sliding between the near and far regions of the upper substrate. If the free-energy correction to the non-nodal atoms in the coarse-graining merging regions is removed, the multiscale method can still well reproduce the shear stress profile, the mean separation curve and the transverse stress profile in the fully atomistic simulation of the system. In addition, when the free-energy-corrected HACG simulation predict successively the macroscopic thermomechanical quantities observed in the fully atomistic simulation, the multiscale method can also generate the microscopic atomistic positions with respect to the fluctuation of those in the fully atomistic simulation.

\section{ACKNOWLEDGMENTS}

One of the authors (Z.-B.W.) thanks the National Science Foundation of China for partial support through the Grants No. 11172310, No. 11472284, and the IMECHCAS SHENTENG1800 and SCCAS SHENTENG-7000 research computing facilities for assisting in the computation.

\section{APPENDIX: COMPUTATIONAL DETAILS OF THERMOMECHANICAL PROPERTIES OF THE SYSTEM BY THE FREE-ENERGY-CORRECTED QC METHOD WITH THE COARSE-GRAINING LOCAL (NONLOCAL) ELEMENTS}

The basic techniques in the free-energy-corrected QC simulation, i.e, the shearing, diffusive, and compression-dilation steps of the system in one MC cycle, are similar to those in the previous dynamically constrained QC simulation [24], except for adding the free-energy contribution of non-nodal atoms in each element given in Eqs. (9), (11), and (12). However, the computational details of thermomechanical properties of the system coarse grained with the local and nonlocal elements have significant differences and are summarized as follows.

For the system coarse grained with the nonlocal elements, first, when the system is sheared, the up wall and the nodes in it move a short distance in the transverse direction. The atoms and the centroid atoms underlying elements related to the moving nodes are displaced. The systematic energy is calculated as the initial one. Second, the diffusive step includes of the moves of nodes in the far regions, atoms in the near regions, and fluid atoms in the thin film. The active nodes in the far regions are randomly displaced one by one. When an active node in the far region is migrated, all atoms underlying the six elements sharing it have corresponding moves. The total energy in the substrate is redetermined due to the renewed elements. If the renewed elements are close to the wall, the energies of the elements, which refer to the interaction of their centroid atoms and the wall, are recalculated. If the renewed elements are in the merging region of the substrate, the interaction of the atoms between the near and far regions is also reconsidered. Moreover, when the atoms in the near region are randomly displaced one by one, their interactions with themselves, the atoms in the far region and fluid atoms in the thin film are redetermined. Furthermore, the fluid atoms have several small and large random moves in one MC cycle to facilitate the equilibrium of the thin film. The moves of the fluid atoms only influence on the interactions with themselves and the atoms in the near region. Third, in the compression-dilation step of the system in the vertical direction, all atoms in the up wall, all nodes and the atoms underlying elements in the far regions, all atoms in the near regions, and all fluid atoms in the thin film are displaced. The systematic energy is also changed.

Similarly, for the system coarse grained with the local elements, the shearing, diffusive and compression-dilation steps of the system in one MC cycle are simplified due to the fact that the centroid atom in each element only interacts with the atoms underlying the element in the cut-off circles.
[1] A. W. Adamson, Physical Chemistry of Surfaces, 3rd ed. (Wiley, New York, 1976), Chap. X.

[2] S. Kohlhoff, P. Gumbsch, and H. F. Fischmeister, Philos. Mag. A 64, 851 (1991).

[3] E. B. Tadmor, M. Ortiz, and R. Phillips, Philos. Mag. A 73, 1529 (1996).

[4] V. Shenoy, V. Shenoy, and R. Phillips, Mater. Res. Soc. Symp. Proc. 538, 465 (1999).

[5] G. S. Smith, E. B. Tadmor, and E. Kaxiras, Phys. Rev. Lett. 84, 1260 (2000).

[6] J. Knap and M. Ortiz, J. Mech. Phys. Solids 49, 1899 (2001).

[7] R. Phillips, M. Dittrich, and K. Schulten, Ann. Rev. Mater. Res. 32, 219 (2002).

[8] W. A. Curtin and R. E. Miller, Model. Simul. Mater. Sci. Eng. 11, R33 (2003).

[9] S. O. Nielsen, C. F. Lopez, G. Srinivas, and M. L. Klein, J. Phys.: Condens. Matter 16, R481 (2004).
[10] R. E. Rudd and J. Q. Broughton, Phys. Rev. B 72, 144104 (2005).

[11] L. M. Dupuy, E. B. Tadmor, R. E. Miller, and R. Phillips, Phys. Rev. Lett. 95, 060202 (2005).

[12] S. P. Xiao and W. X. Yang, Int. J. Comput. Methods 2, 293 (2005).

[13] Z. Tang, H. Zhao, G. Li, and N. R. Aluru, Phys. Rev. B 74, 064110 (2006).

[14] X. Liu and S. Li, J. Chem. Phys. 126, 124105 (2007).

[15] Y. Kulkarni, J. Knap, and M. Ortiz, J. Mech. Phys. Solids 56, 1417 (2008).

[16] B. Eidel, Int. J. Mater. Res. 100, 1503 (2009).

[17] A. Yavari and A. Angoshtari, Int. J. Solids Struct. 47, 1807 (2010).

[18] J. Marian, G. Venturini, B. L. Hansen, J. Knap, M. Ortiz, and G. H. Campbell, Model. Simul. Mater. Sci. Eng. 18, 015003 (2010).

[19] M. Iyer and V. Gavini, J. Mech. Phys. Solids 59, 1506 (2011). 
[20] X. H. Li and M. Luskin, Int. J. Multiscale Comput. Eng. 10, 33 (2012).

[21] S. Ganguly, S. Sengupta, P. Sollich, and M. Rao, Phys. Rev. E 87, 042801 (2013).

[22] Z.-B. Wu, D. J. Diestler, R. Feng, and X. C. Zeng, J. Chem. Phys. 119, 8013 (2003).

[23] D. J. Diestler, Z.-B. Wu, and X. C. Zeng, J. Chem. Phys. 121, 9279 (2004).

[24] Z.-B. Wu, D. J. Diestler, R. Feng, and X. C. Zeng, J. Chem. Phys. 120, 6744 (2004).
[25] Z.-B. Wu, D. J. Diestler, and X. C. Zeng, J. Chem. Phys. 121, 8029 (2004).

[26] Z.-B. Wu, D. J. Diestler, and X. C. Zeng, Mol. Sim. 31, 811 (2005).

[27] R. LeSar, R. Najafabadi, and D. J. Srolovitz, Phys. Rev. Lett. 63, 624 (1989).

[28] M. Schoen, D. J. Diestler, and J. H. Cushman, Phys. Rev. B 47, 5603 (1993).

[29] M. Schoen, S. Hess, and D. J. Diestler, Phys. Rev. E 52, 2587 (1995). 